\title{
BMJ Open Body mass index in school-aged children and the risk of routinely diagnosed non-alcoholic fatty liver disease in adulthood: a prospective study based on the Copenhagen School Health Records Register
}

\author{
Esther Zimmermann, ${ }^{1}$ Michael Gamborg, ${ }^{1}$ Claus Holst, ${ }^{1}$ Jennifer L Baker, ${ }^{1,2}$ \\ Thorkild I A Sørensen, ${ }^{1,2,3}$ Tina L Berentzen ${ }^{1}$
}

To cite: Zimmermann E, Gamborg M, Holst C, et al. Body mass index in schoolaged children and the risk of routinely diagnosed nonalcoholic fatty liver disease in adulthood: a prospective study based on the Copenhagen School Health Records Register. BMJ Open 2015:5:e006998.

doi:10.1136/bmjopen-2014006998

\section{- Prepublication history and additional material is available. To view please visit the journal (http://dx.doi.org/ 10.1136/bmjopen-2014- 006998).}

Received 23 October 2014 Revised 10 April 2015 Accepted 11 April 2015

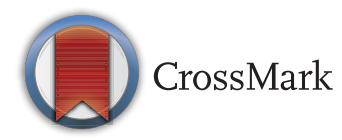

For numbered affiliations see end of article.

\section{Correspondence to} Dr Esther Zimmermann; esther.zimmermann@ regionh.dk

\section{ABSTRACT}

Objective: The relation between childhood overweight and adult non-alcoholic fatty liver disease (NAFLD) is largely unknown. We investigated if weight and weight gain in childhood increases the risk of being diagnosed with NAFLD in routine clinical settings in adulthood.

Participants: We studied 244464 boys and girls, born between 1930 and 1989, who attended school in Copenhagen, Denmark. Their heights and weights were measured by physicians or nurses at mandatory school health examinations at ages 7-13 years. Body mass index (BMI) z-scores were calculated from an internal age-specific and sex-specific reference.

Outcome measures: NAFLD reported in the National Patient Register and the National Register of Pathology at 18 years of age or older. HRs with $95 \% \mathrm{Cls}$ were estimated.

Results: During follow-up, 1264 and 1106 NAFLD cases, respectively, occurred in men and women. In both sexes, childhood BMI z-score was not consistently associated with adult NAFLD. Change in BMI z-score between 7 and 13 years of age was positively associated with NAFLD in both sexes. When adjusted for BMI z-score at age 7 years, the HRs of adult NAFLD were $1.15(95 \% \mathrm{Cl} 1.05$ to 1.26$)$ and $1.12(95 \% \mathrm{Cl} 1.02$ to 1.23$)$ per 1 -unit gain in BMI z-score in men and women, respectively. Associations were similar when adjusted for BMI z-score at age 13 years, and were consistent across birth years.

Conclusions: A BMI gain in school-aged children is associated with adult NAFLD. Intriguingly, BMI gain appears to have an effect on adult NAFLD irrespective of either the initial or the attained BMI. Taken together, our results suggest that BMI gain in childhood, rather than the level of BMI per se, is important in the development of adult NAFLD.

\section{INTRODUCTION}

The obesity epidemic has affected children as well as adults during the past decades, and

\section{Strengths and limitations of this study}

Compared with previously published studies, our sample size and number of cases are much larger. In combination with the long duration of follow-up, this allowed us to thoroughly investigate the relation between childhood body size and the risk of non-alcoholic fatty liver disease (NAFLD) diagnosed in adulthood.

- The children's height and weight were objectively measured by trained nurses and physicians once a year at the mandatory school health examinations.

- A key strength of this study is the focus on the cases that, based on routine clinical practice, resulted in a discharge diagnosis of NAFLD, as this case segment may be the most relevant to study both from a clinical and public health point of view.

- In this large-scale epidemiological study, a more detailed investigation based on the processes of diagnosing NAFLD in the clinical setting is not possible. Furthermore, the data do not allow an investigation of weight changes before or after 7 and 13 years of age.

- We found evidence that a gain in childhood BMI, rather than the level of either initial or attained $\mathrm{BMI}$, is important in the development of NAFLD diagnosed in adulthood.

the prevalence of obesity has never been higher. ${ }^{1-4}$ Alongside this, an epidemic of non-alcoholic fatty liver disease (NAFLD) has emerged. NAFLD is a condition defined by excessive fat accumulation in the liver, and represents a spectrum of varying degrees of liver disease that is not attributable to alcohol or other known causes of liver disease. $^{5}$ It ranges from steatosis, which is 
considered relatively benign, to the more progressive form of non-alcoholic steatohepatitis with varying degrees of fibrosis and eventual cirrhosis. ${ }^{6}$ NAFLD is often associated with insulin resistance and other metabolic alterations, and is considered the hepatic manifestation of the metabolic syndrome. ${ }^{7}$ In the Western world, NAFLD is now the most frequent form of chronic liver disease in children and adults. ${ }^{8-10}$ In the USA, the prevalence of NAFLD may be as high as $30 \%$ among the general population ${ }^{11}$ and it is present in $80-90 \%$ of obese adults. ${ }^{7}$

Evidence is growing that childhood overweight and weight gain may lead to a range of metabolic diseases in adults. ${ }^{12} 13$ We have previously shown that childhood overweight is associated with adult liver cancer. ${ }^{14}$ These findings deserve further exploration, as NAFLD is considered a risk factor for liver cancer. ${ }^{15} \mathrm{~A}$ few studies have found indications that childhood body size is related to a later increased risk of adult NAFLD. ${ }^{16-18}$ However, these studies had small sample-sizes, ${ }^{16}{ }^{18}$ limited length of follow-up ${ }^{16}$ and used NAFLD markers. ${ }^{16}{ }^{18}$ The present study is focused on cases that in routine clinical practice resulted in a discharge diagnosis of NAFLD. Since ascertainment of these cases required hospitalisation, they are likely to have represented the more severe cases. However, from both a clinical and public health point of view, this case segment may be the most important to study, also in relation to childhood BMI as a risk factor. Therefore, the aim of the present study was to investigate the associations between childhood BMI, changes in childhood BMI and routinely diagnosed adult NAFLD.

\section{METHODS}

\section{Study population}

A large cohort was drawn from the Copenhagen School Health Records Register (CSHRR). ${ }^{19}$ The CSHRR is an unselected population consisting of 372636 school children, born between 1930 and 1989, who ever attended school in the Copenhagen municipality. Until 1983, the children had yearly measurements of height and weight at each age between 7 and 13 years as a part of the mandatory school health examinations. Thereafter, the measurements were only taken at school entry and exit, and more frequently if the child had special needs. ${ }^{19}$ Each child had a unique health card that was filled out by the school physicians or nurses who also performed the height and weight measurements. Information on height and weight is now computerised for all 372636 children.

Body mass index (BMI) was calculated for each child at each age, and BMI z-scores were computed from an internal age-specific and sex-specific reference population using the lambda-mu-sigma (LMS) method. ${ }^{20}$ The reference population included children with weight and height data between 1955 and 1960, as the prevalence of obesity was low and stable during this period. ${ }^{12} 19$
Z-scores were interpolated to the exact age if two measurements were available within \pm 1 year around the age of interest or extrapolated if only one measurement was available within a \pm 1 year period. Positive z-scores indicate BMI values above the median, and negative z-scores indicate BMI values below the median in the reference population.

The children were followed prospectively in national registers via their unique personal identification number that was introduced in Denmark on 2 April 1968. ${ }^{21}$ The number was recorded on each child's health card. Children who left school prior to this time, but who were still alive in 1968, had their number retrieved on the basis of information on the name, date and place of birth. Unique personal identification numbers were identified for 329968 (89\%) individuals. ${ }^{19}$

NAFLD cases were determined by linking data from two sources: The Danish National Patient Register ${ }^{22}$ and the Danish National Register of Pathology. ${ }^{23}$ The National Patient Register contains discharge diagnosis for every individual hospitalised in Denmark since 1977. The discharge diagnosis, NAFLD, was made by applying conventional diagnostic tools such as blood tests, various imaging techniques as well as liver biopsy. The NAFLD diagnosis was recorded according to the International Classification of Disease (ICD) eighth revision until 1994 and the 10th revision thereafter (see online supplementary appendix 1). The National Register of Pathology holds information on all liver biopsies performed in hospital departments in Denmark since 1990. In this register, the NAFLD diagnosis was defined according to Systemised NOmenclature of MEDicine (SNOMED versions 2 and 3, Danish extension) codes from 1 January 1990, when electronic registration was introduced in all pathology departments in Denmark (see online supplementary appendix 1). Therefore, we had complete coverage of NAFLD diagnosed in hospital departments from both registers from 1 January 1990.

To restrict the analyses to NAFLD diagnosed in adulthood, the age at entry was set to 18 years. Thus, the follow-up of the individuals began at 18 years of age or on 1 January 1990, whichever came later. NAFLD was defined as the first occurrence of routinely diagnosed non-alcoholic steatosis, steatohepatitis, fibrosis or cirrhosis (whichever came first), following baseline, that is, 18 years of age or on 1 January 1990, and is hereafter denoted 'adult NALFD'. To eliminate a potential mixed aetiology of the adult NAFLD diagnoses, analyses were performed only in individuals free of a previous diagnosis of hepatitis (viral or autoimmune), biliary cirrhosis or alcohol-related disorders (see online supplementary appendix 1).

A total of 20384 individuals were excluded prior to follow-up as they had died $(n=11625)$, emigrated $(n=7613)$, were lost to follow-up $(n=332)$, diagnosed with NAFLD $(n=20)$ or had a diagnosis of an alcohol-related disorder $(\mathrm{n}=165)$, hepatitis (viral or autoimmune; $\mathrm{n}=624)$ or biliary cirrhosis $(\mathrm{n}=5)$ before the age of 
18 years or by 1 January 1990. Therefore, 309584 children were eligible for our analyses, of whom 244464 (49.8\% men) had available BMI values at both age 7 and 13 years, and thus were included in the analyses. Follow-up ended on the date of a NAFLD diagnosis $(n=2370)$, of a diagnosis of hepatitis, biliary cirrhosis or alcohol-related disorders $(n=4282)$, of death $(n=29619)$, of emigration $(n=4485)$, of loss to follow-up $(n=80)$ or 1 January 2010 ( $\mathrm{n}=203$ 628), whichever came first.

\section{Statistical analysis}

Analyses of childhood BMI were performed separately for boys and girls. HRs of adult NAFLD according to BMI z-score at each age from 7 to 13 years were estimated using Cox proportional-hazards regression with age as the underlying time scale. Next, we investigated if change in BMI z-score between the ages of 7 and 13 years was associated with adult NAFLD, using two different approaches. First, we investigated whether, at a certain level of initial BMI, future BMI change was important. That is, we investigated BMI change conditional on initial BMI at age 7 years. Second, we investigated whether, at a certain level of attained BMI, the history of BMI change was important. Accordingly, we investigated BMI change conditioned on attained BMI at age 13 years. To take potential changes in the determinants of adult NAFLD during the study-period into account, all analyses were conducted with the baseline hazard estimated in 5-year strata of year of birth (19301934, 1935-1939,...,1985-1989).

The assumption of linearity on the log HR scale of the continuous variables was tested against a restricted cubic spline (four knots at the 5th, 35th, 65th and 95th centiles). ${ }^{24}$ Since non-linear associations were detected between BMI z-scores at ages 7 through 13 years and adult NAFLD, the effect estimates for these associations were computed via piecewise linear models with a knot point at the median BMI z-score of 0 .

The association between change in BMI z-score and adult NAFLD may depend on the initial and on the attained BMI of the individual. Thus, we examined whether the effect of change was similar in individuals with a BMI below and above a BMI z-score of 0 , at 7 and 13 years of age, respectively. Moreover, we investigated if the associations were dependent on year of birth (5-year-intervals: 1930-1934, 1935-1939,..,1985-1989). All interactions were formally tested on the log HR scale by deviance tests based on comparisons of $-2 \log$ likelihood in nested models with and without cross-product terms. The proportional hazard assumptions were assessed by a test based on Schoenfeld residuals, and no deviations were detected. ${ }^{25}$

\section{Sensitivity analysis}

All analyses were repeated for each of the clinical stages of NAFLD; that is, with steatosis, steatohepatitis, fibrosis and cirrhosis as the outcome (see online supplementary appendix 1).
Alcohol use disorders are among the most prevalent psychiatric disorders in the general population, and individuals with severe alcohol intake, for example, alcoholism, may likely be diagnosed with such a condition in the National Psychiatric Central Register. ${ }^{26}$ To further examine the potential influence of any alcoholic condition on NAFLD aetiology, a set of analyses was therefore conducted in individuals free of any alcohol-related diagnosis in the National Psychiatric Central Register ${ }^{26}$ (see online supplementary appendix 1).

Finally, a shift in the registration of NAFLD occurred in 1994 by the introduction of the ICD-10 coding system, which may have influenced our findings. All analyses were therefore repeated with follow-up from 1994 onwards.

\section{RESULTS}

As expected for children, the mean and the variation in BMI of the boys and girls increased with age (table 1). The follow-up period covered the age range from 18 through 80 years, during which 1264 and 1106 NAFLD cases occurred in men and women, respectively (table 2). The incidence rate of NAFLD increased with advancing age from 0.1 per 1000 person years at 18 years of age to 0.9 per 1000 person years at 80 years of age (figure 1 ). The median age at a NAFLD diagnosis was 54.6 years in men and 55.8 years in women, and men generally had a higher risk of NAFLD than women, with an estimated HR of 1.20 (CI 1.11 to 1.30) for men versus women.

\section{Childhood BMI and risk of adult NAFLD}

The associations between BMI z-score and adult NAFLD were non-linear, especially at the younger ages (table 3 ). Hence, the results are presented for BMI z-scores below and above a z-score of 0 at each age from 7 to 13 years. The HRs for the lower part of the BMI range were elevated, but non-significant, among the boys and decreased with age at measurement. There were no significant associations between the lower part of the BMI range and later NAFLD among girls. The HRs for the upper part of the BMI range were elevated and increased with age at measurement, but remained nonsignificant in both sexes (table 3). No interactions between BMI z-score and year of birth in relation to risk of NAFLD were observed in men or women (all $p$ for interactions $>0.05$ ).

\section{Change in BMI between ages 7 and 13 years, and risk} of adult NAFLD

The mean change in BMI z-score between 7 and 13 years of age was $0.1 \mathrm{BMI} z$-score in boys as well as in girls (table 1). Change in BMI z-score conditional on BMI z-score at age 7 years resulted in HRs of adult NAFLD of 1.15 (95\% CI 1.05 to 1.26$)$ and 1.12 (95\% CI 1.02 to 1.23) per 1-unit gain in BMI z-score between ages 7 and 13 years in men and women (figure $2 \mathrm{~A}$ and $\mathrm{B}$ ). Conditioned on BMI z-score at age 13 years, the HRs of 


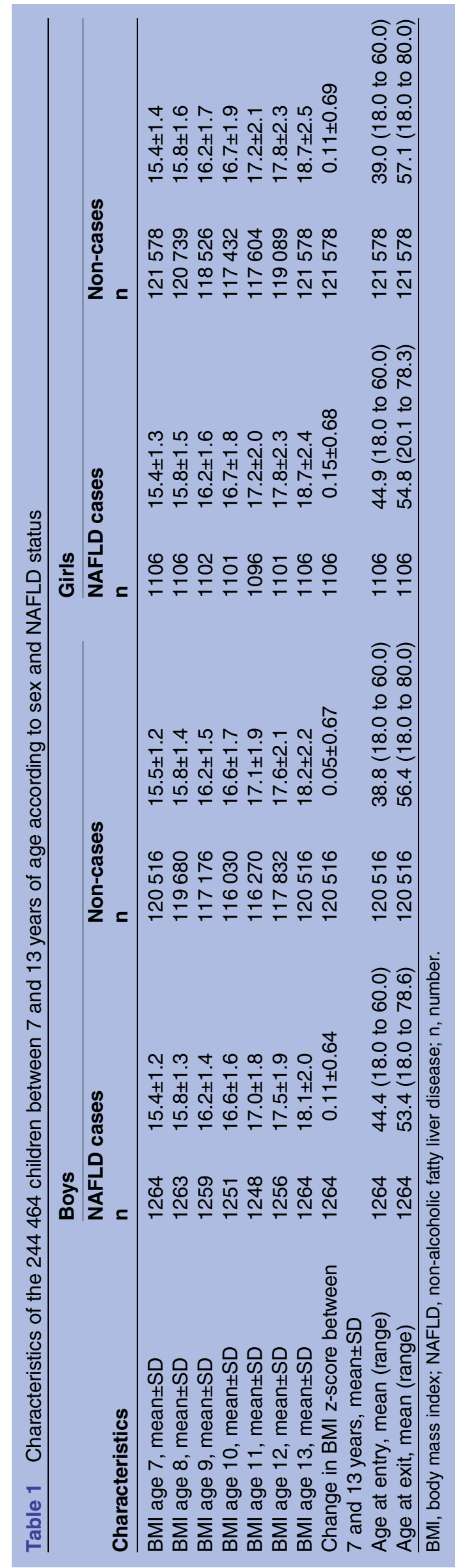

adult NAFLD were 1.16 (95\% CI 1.06 to 1.26 ) and 1.06 (95\% CI 0.97 to 1.16 ) per 1-unit gain in BMI z-score between 7 and 13 years of age in men and women (figure $3 \mathrm{~A}$ and $\mathrm{B}$ ). The above associations were not dependent on the level of BMI at ages 7 or 13 years ( $p$ for interactions $>0.05$ ) .

\section{Sensitivity analysis}

Results for change in BMI z-score between 7 and 13 years of age were essentially similar when the analyses were repeated in each of the clinical stages of steatosis, steatohepatitis, fibrosis and cirrhosis (see online supplementary table S1).

Results were slightly strengthened when the analysis was repeated in the 237196 individuals and 1858 cases free of any alcohol-related diagnosis in the National Psychiatric Central Register at baseline (see online supplementary figures S1 and S2).

Results were essentially similar when the analysis was repeated with follow-up from 1994 onwards (see online supplementary table 2).

\section{DISCUSSION}

This large longitudinal study did not show clear associations between higher childhood BMI and routinely diagnosed adult NAFLD. A gain in BMI between 7 and 13 years of age was positively associated with adult NAFLD when adjusted for initial as well as attained BMI. The associations were consistent within each clinical stage of NAFLD (steatosis, steatohepatitis, fibrosis and cirrhosis), and remained unchanged across year of birth. Taken together, our results imply that gain in BMI in childhood influences the risk of NAFLD, irrespective of either the initial or the attained BMI.

The large size of our study allowed us to investigate the association between childhood body size and adult NAFLD separately in relation to age and sex. The risk estimates of the associations between higher BMI at any age from 7 to 13 years and incidence of adult NAFLD were positive, though non-significant, in men as well as in women. We explored the impact of childhood BMI change on adult NAFLD from two different perspectives. In the analysis that was conditioned on initial BMI, we found that among men with equal BMIs at 7 years of age, the risk of NAFLD increased by $15 \%$ per 1-unit gain in BMI z-score between 7 and 13 years of age. To better illustrate this effect size, we compare two average boys at 7 years of age (BMI of $15.4 \mathrm{~kg} / \mathrm{m}^{2}$ ). One boy is still average at 13 years of age (BMI of $17.8 \mathrm{~kg} / \mathrm{m}^{2}$ ), whereas the other boy has a similar growth in height, but gains an additional $6 \mathrm{~kg}$, which translates into a $15 \%$ increased risk of adult NAFLD. The associations were similar among women. Few of the children in the cohort gained 1 unit BMI z-score during their school-years; nonetheless, the linearity of the associations implies that even a small amount of weight gain increases the risk of adult NAFLD. From the analysis conditioned on initial 
Table 2 Routinely diagnosed non-alcoholic fatty liver disease given as numbers and age at diagnosis

\section{Men}

n

\section{Women}

n
Age (years) at diagnosis given as median ( 5 and 95 centiles)
Age (years) at diagnosis given as median ( 5 and 95 centiles)

\begin{tabular}{cr}
\hline First incidence of non-alcoholic \\
Steatosis & 580 \\
Steatohepatitis & 428 \\
Fibrosis & 193 \\
Cirrhosis & 565 \\
Total NAFLD* & 1264
\end{tabular}

$\begin{array}{lrr}52.8(32.0 \text { to } 69.3) & 606 & 55.8(38.1 \text { to } 68.4) \\ 53.9(30.2 \text { to } 70.8) & 375 & 54.8(30.6 \text { to } 70.5) \\ 55.4(33.4 \text { to } 71.3) & 149 & 54.8(35.6 \text { to } 71.1) \\ 55.6(38.0 \text { to } 70.8) & 373 & 55.8(40.9 \text { to } 70.5) \\ 54.6(32.9 \text { to } 70.3) & 1106 & 55.8(36.1 \text { to } 70.3)\end{array}$

${ }^{*}$ The total number of non-alcoholic steatosis, steatohepatitis, fibrosis and cirrhosis is larger than the total number of NAFLD cases. The numbers of events are calculated as first incidence, thus, if an individual has more than one of the NAFLD subdiagnoses, he/she will appear in more than one of the NAFLD subcategories.

$\mathrm{n}$, number; NAFLD, non-alcoholic fatty liver disease.

BMI, we cannot determine whether BMI gain is a risk factor for adult NAFLD independent of attained BMI. Therefore, we also investigated change in BMI between ages 7 and 13 years conditioned on BMI at age 13 years, and found essentially the same results. Thus, although the children end up with equal BMIs at age 13 years, the history of BMI change remains an independent risk factor for adult NAFLD. These results suggest that a gain in childhood BMI has a larger impact on adult NAFLD than initial BMI and that it affects adult NAFLD beyond its effect of attained childhood BMI.

Our findings add to three previous studies on childhood body size and markers of adult NAFLD. ${ }^{16-18}$ A study from Australia found positive associations between different adiposity measures from age 3 to 17 years and ultrasound determined NAFLD at age 17 years. ${ }^{17}$ A study from Finland found positive associations between body size in early childhood and adult liver fat score at age 60 years, but the association became inverse when adjusted for concurrent BMI at that age. ${ }^{18}$ In other

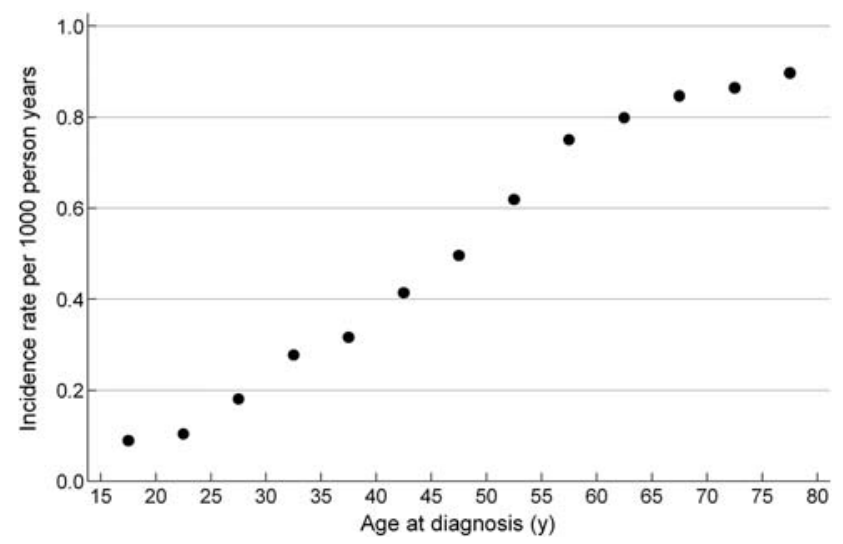

Figure 1 Incidence rate of routinely diagnosed adult non-alcoholic fatty liver disease (NAFLD) per 1000 person years according to age at diagnosis. The incidence rates of routinely diagnosed NAFLD was estimated as the number of individuals registered with a diagnosis of NAFLD for the first time divided by the number of person years at risk in 5-year-age-intervals. words, this study compared individuals with similar adult BMI, but different body size in childhood, and found that the individuals who had the smallest body size as a child had the largest risk of fatty liver in adulthood. One explanation may be that the individuals with the smallest body size in childhood for given weight in adulthood also are those with the greatest weight gain between childhood and adulthood. Similarly, positive associations between childhood adiposity measures and NAFLD, determined by ultrasound and liver enzymes at age 18 years, were reported in a UK study; however, when adjusted for fat mass at 18 years of age these associations were attenuated towards the null. ${ }^{16}$ A major difference between these previous studies and the present study is that they examined the prevalence of NAFLD, assessed as degree of fatty liver, at the same time as the last body weight assessment, whereas we investigated the incidence of routinely diagnosed NAFLD, many years after the last body weight assessment at 13 years of age. Furthermore, these previous studies all used ultrasound-determined fatty liver, and had the possibility for adjustment for not only concurrent body weight, but also alcohol use and other pertinent covariates at the time of fatty liver diagnosis. Our sample size was much larger, and we had access to data on all stages of clinically diagnosed NAFLD, but our study design did not allow adjustment for possible confounders, such as physical activity, various dietary factors and smoking. Based on our findings, we speculate that a weight gain during school-years may induce an increased susceptibility for developing NAFLD, including also the severe stages of the NAFLD complex, later in life.

A unique feature of our study is that we have histological data on each of the clinical stages of NAFLD. Cirrhosis represents the end-stage of liver pathology and it significantly impacts overall and liver-related mortality. ${ }^{27}$ Interestingly, we found that among men with similar attained BMIs at 13 years of age, those who gained in BMI had a $16 \%$ increased risk of adult cirrhosis per 1-unit gain in BMI z-score. Why excessive weight gain in childhood seems to influence the entire spectrum of adult NAFLD is not known. One explanation may be that children with a BMI gain are on the way to becoming 
Table $3 \mathrm{HR}$ and $95 \% \mathrm{Cls}$ for the risk of routinely diagnosed NAFLD in adulthood in relation to a 1-unit lower BMl z-score below and a 1-unit higher BMI Z-score above the median BMI Z-score in a cohort of 244464 children

\section{HR $(95 \% \mathrm{Cl}$ ) in relation to BMI z-score below (BMI z-score $<0$ ) and above the median (BMI z-score $>0$ )}

\begin{tabular}{llllll}
\hline Age & Boys & Below & Above & & Girls \\
\hline 7 & $1.12(1.00$ to 1.23$)$ & & Below & Above \\
8 & $1.10(0.99$ to 1.23$)$ & $1.02(0.91$ to 1.16$)$ & & $0.96(0.85$ to 1.08$)$ & $0.99(0.88$ to 1.12$)$ \\
9 & $1.11(1.00$ to 1.24$)$ & $1.07(0.95$ to 1.20$)$ & & $1.00(0.88$ to 1.13$)$ & $1.03(0.91$ to 1.17$)$ \\
10 & $1.08(0.96$ to 1.21$)$ & $1.08(0.96$ to 1.22$)$ & & $0.96(0.84$ to 1.13$)$ & $1.05(0.92$ to 1.18$)$ \\
11 & $1.07(0.95$ to 1.20$)$ & $1.09(0.97$ to 1.23$)$ & & $0.94(0.83$ to 1.08$)$ & $1.03(0.91$ to 1.17$)$ \\
12 & $1.03(0.92$ to 1.16$)$ & $1.05(0.93$ to 1.18$)$ & & $0.98(0.86$ to 1.11$)$ & $1.04(0.92$ to 1.18$)$ \\
13 & $1.04(0.92$ to 1.17$)$ & $1.10(0.98$ to 1.24$)$ & & $0.96(0.84$ to 1.09$)$ & $1.07(0.95$ to 1.21$)$ \\
\hline
\end{tabular}

The HRs illustrate the risk associated with each 1-unit difference in BMI z-score away from zero. For example, a HR of 1.12 (Cl 1.00 to 1.23) in 7 year old boys with a BMI z-score below the median means that the risk of adult NAFLD increases by $12 \%$ per 1 -unit lower BMI z-score below zero. A HR of 1.09 (Cl 0.97 to 1.22) in 13-year-old girls with a BMI z-score above the median means that the risk of adult NAFLD increases by $9 \%$ per 1 -unit higher BMI z-score above zero.
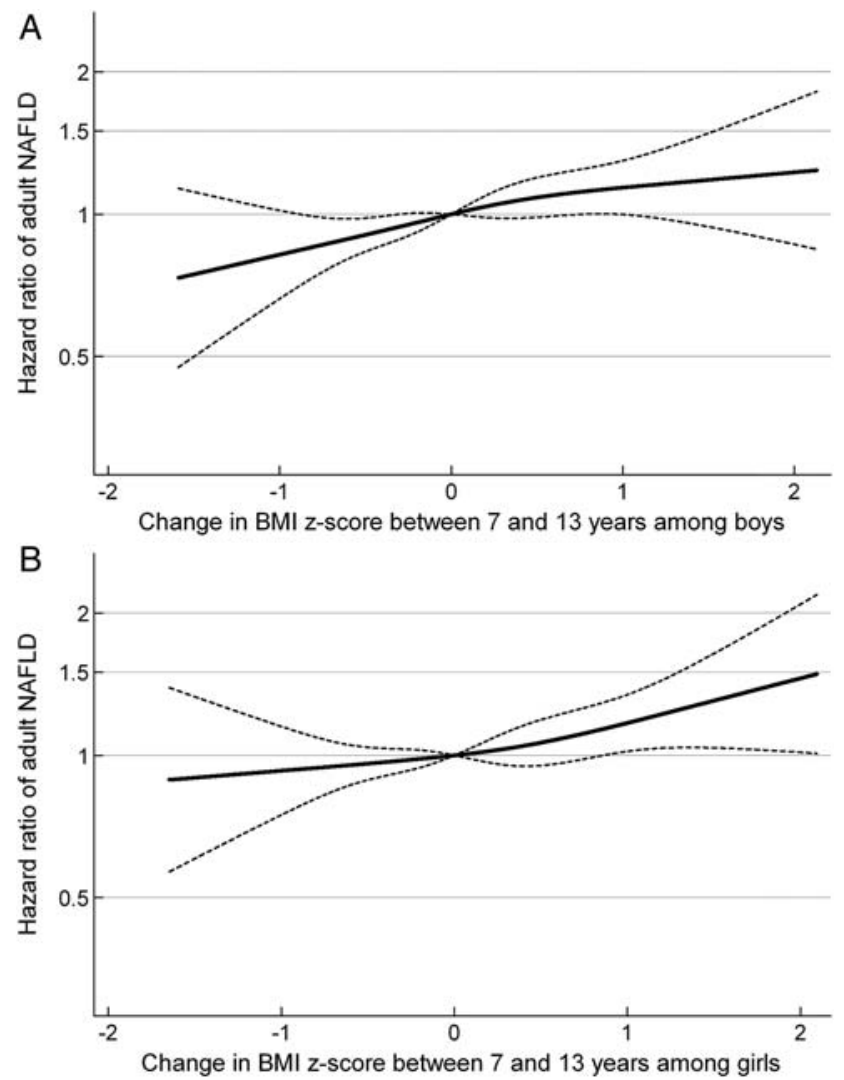

Figure $2 \mathrm{HRs}$ and $95 \% \mathrm{Cl}$ according to change in BMI z-score between the ages of 7 and 13 years, adjusted for BMI $z$-score at 7 years of age, in relation to routinely diagnosed NAFLD in adulthood for (A) boys and (B) girls. (A) Boys: HR of adult NAFLD per 1-unit increase in BMI Z-score between 7 and 13 years of age among boys: $1.15(95 \% \mathrm{Cl} 1.05$ to 1.26$)$. (B) Girls: HR of adult NAFLD per 1-unit increase in BMI z-score between 7 and 13 years of age among girls: 1.12 $(95 \% \mathrm{Cl} 1.02$ to 1.23$)$. The associations are adjusted for BMI z-score at 7 years of age and stratified by birth cohort. The HRs in figure 2 are estimated by Cox regression including change in body mass index (BMI) z-score between the ages of 7 and 13 years, as a restricted cubic spline, with a reference point in change in BMI $\mathrm{z}$-score equal to 0 , and truncated to depict the inner $99 \%$ part of the distribution. NAFLD, non-alcoholic fatty liver disease.
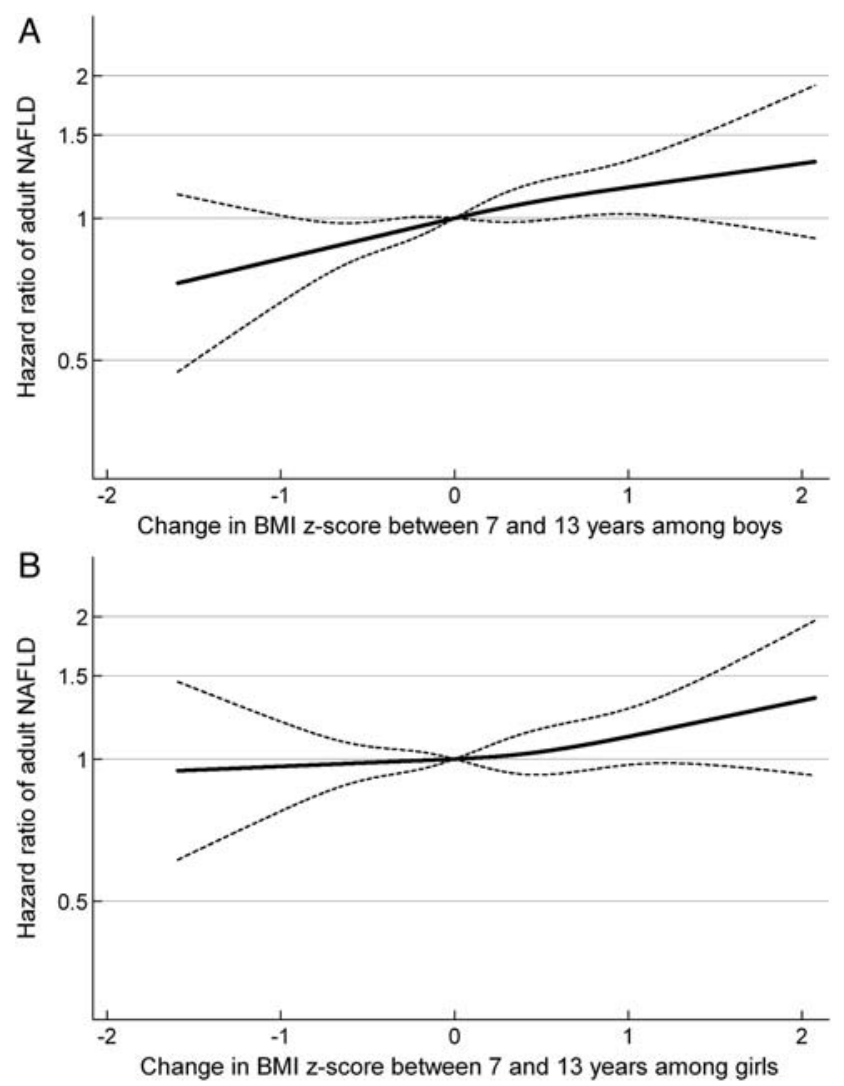

Figure $3 \mathrm{HRs}$ and $95 \% \mathrm{Cl}$ according to change in $\mathrm{BMI}$ z-score between the ages of 7 and 13 years, adjusted for BMI $z$-score at 13 years of age, in relation to routinely diagnosed NAFLD in adulthood for (A) boys and (B) girls. (A) Boys: HR of adult NAFLD per 1-unit increase in BMI z-score between 7 and 13 years of age among boys: $1.16(95 \% \mathrm{Cl} 1.06$ to 1.26$)$. (B) Girls: HR of adult NAFLD per 1-unit increase in BMI z-score between 7 and 13 years of age among girls: 1.06 $(95 \% \mathrm{Cl} 0.97$ to 1.16$)$. The associations are adjusted for BMI z-score at 13 years of age and stratified by birth cohort. The HRs in figure 3 are estimated by Cox regression including change in body mass index (BMI) z-score between the ages of 7 and 13 years, as a restricted cubic spline, with a reference point in change in BMI z-score equal to 0 and truncated to depict the inner $99 \%$ part of the distribution. NAFLD, non-alcoholic fatty liver disease. 
obese adults, and that it is the adult obesity and its associated metabolic complications that increase the risk of adult NAFLD. Although childhood and adult obesity are correlated, the tracking is generally moderate in magnitude. ${ }^{28} 29$ This indicates that other mechanisms are also at play. Several pathways, in particular related to metabolic dysfunctions, have been proposed to explain the progressive liver pathologies associated with obesity. ${ }^{30}$ Such pathways could possibly be accelerated by excessive weight gain in childhood, where the liver may be more sensitive to metabolic derangements. Accordingly, our results may be of biological relevance, irrespective of adult obesity and suggest that prevention of excessive weight gain during childhood may contribute to reducing the rapidly rising incidence of adult NAFLD.

Key advantages of the present study are the large sample size and its prospective design. Our cohort was essentially drawn from all school children in Copenhagen who were born between 1930 and 1989. The attrition in sample size may not induce bias, since it was due to missing data on the children's height and weight measurements, which likely were missing at random. On the other hand, an important limitation is that we do not have height and weight data before or after the ages of 7 and 13 years. Owing to the free access to healthcare in Denmark, the continuous updating of the health registers and the minimal loss to follow-up, it is unlikely that selection bias would have influenced our results. The NAFLD diagnoses from the National Register of Pathology were based on liver biopsies performed by clinical pathologists, reducing the risk of misclassification of NAFLD. By further adding data from the National Patient Register, we achieved the best possible coverage of the routinely diagnosed NAFLD. However, as the subjects in this cohort had to undergo liver biopsy or hospitalisation to be registered as NAFLD cases, we have underestimated the true incidence of NAFLD. This underestimation could have biased the estimated HRs in either direction. Moreover, we have no way of verifying if and how the practice guidelines ${ }^{31}$ for clinicians, when diagnosing NAFLD, were applied. The fact that the results were consistent across the NAFLD spectrum supports the fact that misclassification of events is unlikely to explain our findings. Nevertheless, differential misclassification could bias our results, as it is possible that children with a high BMI may be more likely to be examined for adult NAFLD than children with normal BMI. Information bias is unlikely since the height and weight measurements of the children were performed by trained healthcare personnel years before and thus independent of whether the children were later diagnosed with NAFLD. Finally, nearly a fifth of the NAFLD cases were excluded when data on alcohol-related diagnosis were linked via the National Psychiatric Central Register, which indicates that we cannot fully eliminate an admixture of alcoholic fatty liver disease among the NAFLD cases. On the other hand, the clinicians, having the choice between alcoholic fatty liver disease and NAFLD, have chosen to make the diagnosis NAFLD. We did not have access to information in the patient registers on moderate alcohol use, but the associations between BMI in childhood and later risk of NAFLD were slightly strengthened when individuals with any alcohol-related diagnosis in the National Psychiatric Central Register were excluded and censored. Thus, had we been able to also identify and exclude those with even a slight overuse of alcohol, the associations observed would likely have been even stronger.

In conclusion, excessive BMI gain between the ages of 7 to 13 years was positively associated with the risk of routinely diagnosed adult NAFLD in men as well as in women. Intriguingly, a gain in childhood BMI was a predictor of adult NAFLD irrespective of either the initial or the attained BMI. All together, our results suggest that BMI gain in childhood, rather than body size per se, is important in the development of adult NAFLD.

\section{Author affiliations \\ ${ }^{1}$ Institute of Preventive Medicine, Bispebjerg and Frederiksberg Hospital, The Capital Region, Copenhagen, Denmark \\ ${ }^{2}$ Section on Metabolic Genetics, Faculty of Health and Medical Sciences, Novo Nordisk Foundation Centre for Basic Metabolic Research, University of Copenhagen, Denmark \\ ${ }^{3}$ MRC Integrative Epidemiology Unit, Bristol University, Bristol, UK}

Contributors The study was conceived by $\mathrm{CH}$, TLB and TIAS. The study was designed by EZ with input from MG, CH, TIAS, JLB and TLB. EZ carried out statistical analyses and wrote the first draft of the manuscript. All authors contributed to critical revisions of the manuscript and approved the final submitted version.

Funding This work was carried out as a part of a project supported by the European Union: FLIP (Fatty Liver Inhibition of Progression) under the Seventh Framework Programme (FP7/2007-2013, grant agreement $n^{0}$ Health-F2-2009-241762). This work was also part of the research activities of the Danish Obesity Research Centre (DanORC, see http://www.danorc.dk/), supported by the Danish Council for Strategic Research. The research leading to these results also received funding from the European Research Council under the European Union's Seventh Framework Programme (FP/2007-2013)/ ERC Grant Agreement number 281419, childgrowth2cancer to JLB. The funders had no role in the design or conduct of the study or the decision to publish.

Competing interests None declared.

Ethics approval The present analysis was conducted on anonymous data and it was approved by the Danish Data Protection Agency (Datatilsynet). According to the Danish Act of Processing of Personal data (Persondataloven), informed consent is not required for register-based research of pre-existing personal data.

Provenance and peer review Not commissioned; externally peer reviewed.

Data sharing statement Statistical code can be requested from the corresponding author. For details of data access and collaboration policy, please contact Thorkild I A Sørensen: thorkild.ingvor.arrild. soerensen@regionh.dk

Open Access This is an Open Access article distributed in accordance with the Creative Commons Attribution Non Commercial (CC BY-NC 4.0) license, which permits others to distribute, remix, adapt, build upon this work noncommercially, and license their derivative works on different terms, provided the original work is properly cited and the use is non-commercial. See: http:// creativecommons.org/licenses/by-nc/4.0/

\section{REFERENCES}

1. Flegal KM, Carroll MD, Ogden CL, et al. Prevalence and trends in obesity among US adults, 1999-2008. JAMA 2010;303:235-41. 
2. Ogden CL, Carroll MD, Kit BK, et al. Prevalence of childhood and adult obesity in the United States, 2011-2012. JAMA 2014;311:806-14

3. Popkin BM, Conde W, Hou N, et al. Is there a lag globally in overweight trends for children compared with adults? Obesity (Silver Spring) 2006;14:1846-53.

4. Rokholm B, Baker JL, Sørensen TIA. The levelling off of the obesity epidemic since the year 1999-a review of evidence and perspectives. Obes Rev 2010;11:835-46.

5. McCullough AJ. Update on nonalcoholic fatty liver disease. J Clin Gastroenterol 2002;34:255-62.

6. Kleiner DE, Brunt EM, Van NM, et al. Design and validation of a histological scoring system for nonalcoholic fatty liver disease. Hepatology 2005;41:1313-21.

7. Bellentani S, Scaglioni F, Marino M et al. Epidemiology of non-alcoholic fatty liver disease. Dig Dis 2010;28:155-61.

8. Angulo P. Nonalcoholic fatty liver disease. $N$ Engl J Med 2002;346:1221-31.

9. Anstee QM, Targher G, Day CP. Progression of NAFLD to diabetes mellitus, cardiovascular disease or cirrhosis. Nat Rev Gastroenterol Hepatol 2013;10:330-44.

10. Cheung CR, Kelly DA. Non-alcoholic fatty liver disease in children. BMJ 2011;343:d4460.

11. Lazo M, Clark JM. The epidemiology of nonalcoholic fatty liver disease: a global perspective. Semin Liver Dis 2008;28:339-50.

12. Baker JL, Olsen LW, Sørensen TIA. Childhood body-mass index and the risk of coronary heart disease in adulthood. $N$ Engl J Med 2007;357:2329-37.

13. Park $\mathrm{MH}$, Sovio U, Viner RM, et al. Overweight in childhood, adolescence and adulthood and cardiovascular risk in later life: pooled analysis of three British birth cohorts. PLOS ONE 2013;8:e70684.

14. Berentzen TL, Gamborg M, Holst $\mathrm{C}$, et al. Body mass index in childhood and adult risk of primary liver cancer. J Hepatol 2014;60:325-30.

15. Michelotti GA, Machado MV, Diehl AM. NAFLD, NASH and liver cancer. Nat Rev Gastroenterol Hepatol 2013;10:656-65.

16. Anderson EL, Howe LD, Fraser A, et al. Weight trajectories through infancy and childhood and risk of non-alcoholic fatty liver disease in adolescence: the ALSPAC study. J Hepatol 2014;61:626-32.

17. Ayonrinde OT, Olynyk JK, Marsh JA, et al. Childhood adiposity trajectories and risk of nonalcoholic fatty liver disease in adolescents. J Gastroenterol Hepatol 2015;30:163-71.
18. Sandboge S, Perala MM, Salonen MK, et al. Early growth and non-alcoholic fatty liver disease in adulthood-the NAFLD liver fat score and equation applied on the Helsinki Birth Cohort Study. Ann Med 2013;45:430-7.

19. Baker JL, Olsen LW, Andersen I, et al. Cohort profile: the Copenhagen School Health Records Register. Int J Epidemiol 2009;38:656-62.

20. Cole TJ, Green PJ. Smoothing reference centile curves: the LMS method and penalized likelihood. Stat Med 1992;11:1305-19.

21. Pedersen CB, Gotzsche H, Moller JO, et al. The Danish Civil Registration System. A cohort of eight million persons. Dan Med Bull 2006;53:441-9.

22. Andersen TF, Madsen M, Jorgensen J, et al. The Danish National Hospital Register. A valuable source of data for modern health sciences. Dan Med Bull 1999;46:263-8.

23. Bjerregaard B, Larsen OB. The Danish Pathology Register. Scand $J$ Public Health 2011;39:72-4.

24. Harrell F. Relaxing linearity assumption for continuous predictors. In: Regression modeling strategies: with applications to linear models, logistic regression, and survival analyses. New York: Springer, 2002:16-26.

25. Schoenfeld D. Chi-squared goodness of fit tests for the proporiona hazards regression model. Biometrica 1980;67:145-53.

26. Mors O, Perto GP, Mortensen PB. The Danish Psychiatric Central Research Register. Scand J Public Health 2011;39:54-7.

27. Sun B, Karin M. Obesity, inflammation, and liver cancer. J Hepatol 2012;56:704-13.

28. Singh AS, Mulder C, Twisk JW, et al. Tracking of childhood overweight into adulthood: a systematic review of the literature. Obes Rev 2008;9:474-88.

29. Sørensen TIA, Sonne-Holm S. Risk in childhood of development of severe adult obesity: retrospective, population-based case-cohort study. Am J Epidemiol 1988;127:104-13.

30. Cohen JC, Horton JD, Hobbs $\mathrm{HH}$. Human fatty liver disease: old questions and new insights. Science 2011;332:1519-23.

31. Chalasani N, Younossi Z, Lavine JE, et al. The diagnosis and management of non-alcoholic fatty liver disease: practice Guideline by the American Association for the Study of Liver Diseases, American College of Gastroenterology, and the American Gastroenterological Association. Hepatology 2012;55:2005-23. 